\title{
The Planck Compact Source Catalogues: present and future
}

\author{
Marcos López-Caniego ${ }^{1}$, on behalf of the Planck Collaboration ${ }^{2}$ \\ ${ }^{1}$ European Space Agency, ESAC, Planck Science Office, Camino bajo del Castillo, s/n, \\ Urbanización Villafranca del Castillo, Villanueva de la Cañada, Madrid, Spain \\ email: Marcos.Lopez.Caniego@sciops.esa.int \\ ${ }^{2}$ http:// www.cosmos.esa.int/web/planck/planck-collaboration
}

\begin{abstract}
The Planck Collaboration has produced catalogues of radio and sub-millimeter compact sources at the nine Planck frequencies in total intensity and polarization. In particular, the 2015 Second Planck Catalogue of Compact Sources (PCCS2) contains over 45.000 sources detected in the Planck full mission maps. Since the Planck instruments have polarization capabilities in seven of its nine detectors, we were able to measure the polarized flux density of over 600 sources between 30 and $353 \mathrm{GHz}$. But we are searching not only for compact sources in single frequency maps, and we take advantage of the large frequency coverage of Planck to search for objects with specific emission laws. This is the case of the SZ catalogue of cluster of galaxies (PSZ2), that lists 1653 clusters, 1203 of which are confirmed clusters with clear associations in external data-sets, and the Galactic cold clump catalogue (PGCC) with 13188 objects. The Planck Collaboration has also published a list of high-redshift source candidates (see the report by Ludovic Montier here). These objects are rare bright sub-millimeter sources with an spectral energy distribution peaking between 353 and $857 \mathrm{GHz}$, and have been detected combining Planck and IRAS data. The colours of most of these objects are consistent with redshifts $z>2$, a fraction of which could be lensed objects with redshifts between 2 and 4 .

But new catalogues are foreseen. A multi-frequency compact source catalogue is being produced selecting sources at radio frequencies and studying them across all Planck bands. Multifrequency catalogues can be difficult to produce in experiments like Planck that have a large frequency coverage and very different resolutions across bands. In some cases, a source can be very bright across the whole Planck frequency range and it is easy to do the associations across channels. However, it frequent to find unrelated sub-millimeter sources within the half-degree beam of the $30 \mathrm{GHz}$ low frequency detector, and the association work must be done with great care. For this purpose, we are combining a multi-frequency detection procedure with a principal component analysis to produce the catalogue. In addition, for those sources where a clear identification can be made, we will attempt to include flux density information from Herschel and other experiments, in particular for those blazars that are bright in radio, sub-mm and even in gamma-ray frequencies, as seen by Fermi. Moreover, Planck has made available to the community the single survey frequency maps that allow astronomers to study the long-term variability of their favourite sources. New functionalities will be also added to the Planck Legacy Archive $\dagger$, for example a timeline-cutting tool that will allow one to produce full-sky maps from the Planck timelines for specific periods of time allowing for short-term variability studies of compact sources (e.g., flares). The unique frequency coverage of Planck make these catalogues very valuable for other experiments using the Planck compact source catalogues. For example, experiments like QUIJOTE use Planck selected sources to study the impact of polarized radio source emission on their cosmological fields and other CMB experiments will use Planck polarized compact source information for calibration.
\end{abstract}

Keywords. cosmic microwave background, radio continuum: galaxies, submillimeter, infrared: galaxies. 